\title{
Status of the CMS Pixel project
}

\author{
Lorenzo Uplegger ${ }^{1}$ \\ Fermi National Laboratory
}

(CMS Collaboration)

\begin{abstract}
The Compact Muon Solenoid Experiment (CMS) will start taking data at the Large Hadron Collider (LHC) in 2008. The closest detector to the interaction point is the silicon pixel detector which is the heart of the tracking system. It consists of three barrel layers and two pixel disks on each side of the interaction point for a total of 66 million channels. Its proximity to the interaction point means there will be very large particle fluences and therefore a radiation tolerant design is necessary. The pixel detector will be crucial to achieve a good vertex resolution and will play a key role in pattern recognition and track reconstruction. The results from test beam runs prove that the expected performances can be achieved. The detector is currently being assembled and will be ready for insertion into CMS in early 2008. During the assembly phase a thorough electronic test is being done to check the functionality of each channel to guarantee the performance required to achieve the physics goals. This report will present the final detector design, the status of the production as well as results from test beam runs to validate the expected performance.
\end{abstract}

\section{Introduction}

The Compact Muon Solenoid (CMS) general purpose detector [1] at the Large Hadron Collider (LHC) at CERN, has an all-silicon tracking system. The innermost layers of the tracking detector consists of three Barrel layers and four Forward disks, sitting in a 4 Tesla magnetic field, whose basic building blocks are highly segmented silicon sensors (pixels) tightly coupled with their corresponding PSI46V2 [2] readout chips (ROCs). The pixel cell size of $150 \times 100 \mu \mathrm{m}^{2}$ has been chosen to provide high-resolution $3 \mathrm{D}$ space points close to the interaction point for precise vertex determination and fine granularity for charged track pattern recognition. The pixel detector shown in Fig. 1 covers a pseudo rapidity range $-2.5<\eta<2.5$ matching the acceptance of the central tracker. The pixel detector is essential for the reconstruction of secondary vertices and to form seed tracks for pattern recognition in the tracker. The proximity of the detector to the interaction point required a radiation tolerant design. The sensors are designed to withstand the expected doses of $3 \times 10^{14} \mathrm{neq} / \mathrm{cm} 2 /$ year of nominal LHC operation. An "n-on-n" design with $\mathrm{n}+$ implants [3] was chosen. The readout chip, bump bonded to the sensor has been designed using the $0.25 \mu \mathrm{m}$ CMOS radiation hard technology. Extensive studies have been done in several testbeams to guarantee high efficiencies for highly irradiated detectors.

Currently the detector is under construction in different location around the world and each component is undergoing a series of tests to certify that the quality required will be achieved. Results from these tests shows that the final detector will have $99.9 \%$ good channels.

\section{Pixel System}

The pixel detector consists of three barrel layers (BPix) with two endcap disks (FPix) on each side of the BPix, as shown in Fig. 1.

The $53 \mathrm{~cm}$ long BPix layers will be located at mean radii of 4.4, 7.3 and $10.2 \mathrm{~cm}$. Each layer is made up of ladders and each ladder is made up of eight modules. Each full detector module has dimensions of $1.62 \times 6.63 \mathrm{~cm}^{2}$ and is connected to sixteen ROCs.

The four forward disks consist of twenty-four double sided blades arranged in a fan like structure with a $20^{\circ}$ tilt to induce charge sharing between pixels maximizing the Lorentz drift since now the drift direction is not parallel to the magnetic field. Each blade in a disk has four detector modules, called plaquettes, on the side facing the interaction region, and three plaquettes on the other side

${ }^{1}$ E-mail: uplegger@fnal.gov 
facing away. These plaquettes range in size from two to ten ROCs and are placed so that there is full coverage with the gaps from one side covered by the plaquettes on the other.

\section{Sensors and Electronics}

The sensors are "n-on-n" with n+ implants on n bulk silicon. Electron collection has the advantage that after irradiation induced space charge sign inversion, the highest electric field is located close to the collecting electrodes. Therefore n-on-n sensors can be operated after type inversion, but require inter-pixel isolation. BPix and FPix have adopted a moderated p-spray [3] and optimized p-stop isolation [5] respectively. All sensors are already in hand from the vendors. For the BPix detector they are bump bonded at PSI using indium bumps while FPix sensors are bump bonded at two different vendors using PbSn bumps. Guard rings, at the edge of the sensors, have been designed to operate up to bias voltages of $600 \mathrm{~V}$ before breakdown. The track detection efficiency was measured for both technologies. After a fluence of $8 \times 10^{14} \mathrm{neq} / \mathrm{cm}^{2}$, above the goal of the CMS TDR, the detection efficiency is greater than $99 \%$ both for BPix and FPix.

The ROCs are made in $0.25 \mathrm{~mm}$ CMOS radiation tolerant technology [2]. The $80 \times 52$ pixel cells of a ROC are organized in double columns with buffers for data and timestamps, 32 and 12 words depth respectively. The readout is analog with zero suppression and the row and column information is encoded on six analog levels as shown in Fig. 2.

\section{Pixel Detector Performance}

The design allows for three pixel hits per track up to $|\eta|$ of $\sim 2.5$; providing:

- $\quad$ seeds for pattern recognition;

- $\quad$ precision vertexing near the Interaction Point (IP);

- fast tracking and vertexing in the High Level Trigger (HLT) using only the pixel information [6]

The average two-pixel cluster resolution is better than $10 \mu \mathrm{m}$ in the $100 \mu \mathrm{m}$ pitch direction and better than $15 \mu \mathrm{m}$ in the $150 \mu \mathrm{m}$ direction. These resolutions have been confirmed in a recent test beam.

\section{Pixel Detector Production}

The Barrel detector is produced, tested and commissioned at the Paul Scherrer Institut (PSI). The modules, see Fig. 3, are produced at a rate of six full modules per day, then thoroughly tested and stored in dry boxes, ready to be mounted on the carbon fiber support structure once the module production was complete.

The CMS forward pixel detector construction takes place at various institutions around the world, but the final step in testing and assembly (before it is transported to CERN) occurs at Fermilab's Silicon Detector Facility (SiDet). The read-out chips (ROCs) are bump-bonded to silicon sensors called modules, by outside vendors and these modules are assembled into plaquettes, see Fig. 3, at Purdue University, where quick functionality tests are performed before shipping to SiDet. After a visual inspection, modules undergo a two-day thermal cycling process consisting of ten cycles between +20 and $-15^{\circ} \mathrm{C}$. At this point a detailed characterization at $-15^{\circ} \mathrm{C}$ is performed, since the detector will operate at cold temperature to minimize the effects of radiation damage. They are then mounted on the final support structure, see Fig. 4, quickly tested, and then shipped to CERN.

\section{Current Status}

As of the writing of these proceedings, the construction of both the forward and barrel detectors is approximately $90 \%$ completed. All the sensors and readout chips are in hand and all the barrel modules and forward plaquettes have been built and tested. Barrel modules are being assembled on the carbon fiber structure while $75 \%$ of the half disks have been already shipped to CERN to be tested before the final installation.

This year, several tests have been done to guarantee that the integration and operation of the final Forward Pixel detector will be smooth. A successful integration test into the strip tracker has been performed in July where mechanics and electronic interference have been checked. In parallel to this effort, we also electronically tested a similar subsystem at Fermilab in a magnetic field (B) of $4 \mathrm{~T}$ to see its performance with and without the B field. No effect of B field was found on the electronic or mechanical integrity of the detector. 
We are currently on schedule to install the pixel detector into CMS for the 2008 physics run of the LHC.

\section{Bibliography}

[1] "CMS Tracker Technical Design Report”, The CMS Collaboration, CERN/LHCC 1998-6

[2] "Design and performance of the CMS pixel detector readout chip", H.Chr. Kaestli et al., NIM A565 (2006) 188

[3] "Sensor development for the CMS pixel detector", G. Bolla et al., NIM A485 (2002) 89.

[4] "Position dependence of charge collection in prototype sensors for the CMS pixel detector", T. Rohe et al., IEEE Trans.Nucl.Sci.51 (2004) 1150-1157

[5] "Silicon sensors development for the CMS pixel system”, K. Arndt et al., NIM A511, (2003) 106

[6] “The TriDAS Project, Technical Design Report: Data Acquisition and High-Level Trigger", CMS Collaboration, CERN-LHCC-02-26 (2006).

\section{Pictures}

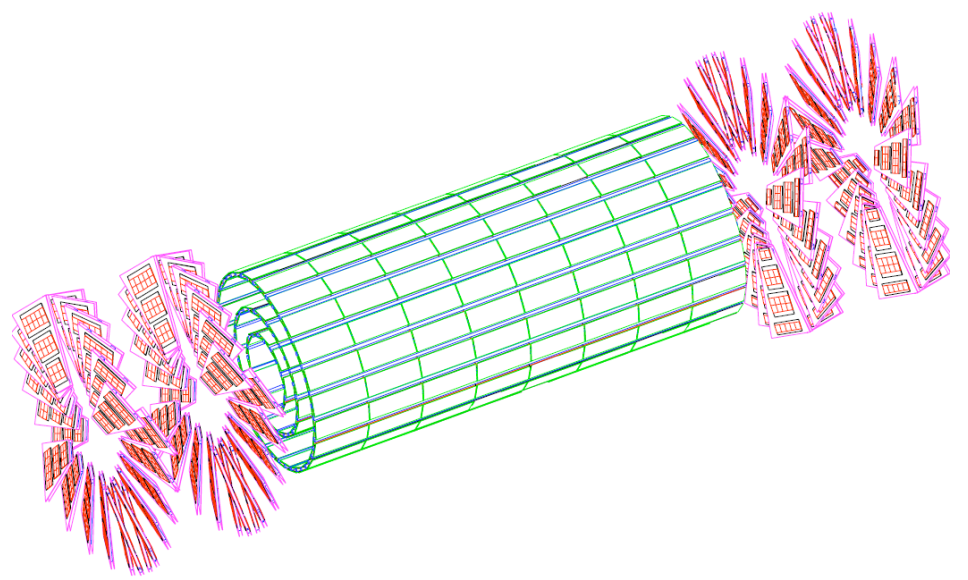

Fig. 1 Schematic view of the CMS pixel detector

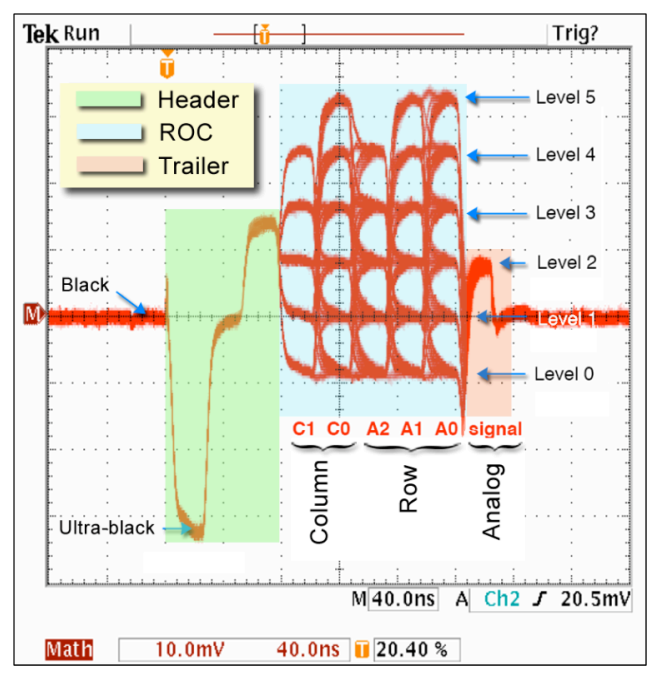

Fig. 2 Analog output of the pixel front-end readout electronics. Columns and rows are encode on six analog levels. 


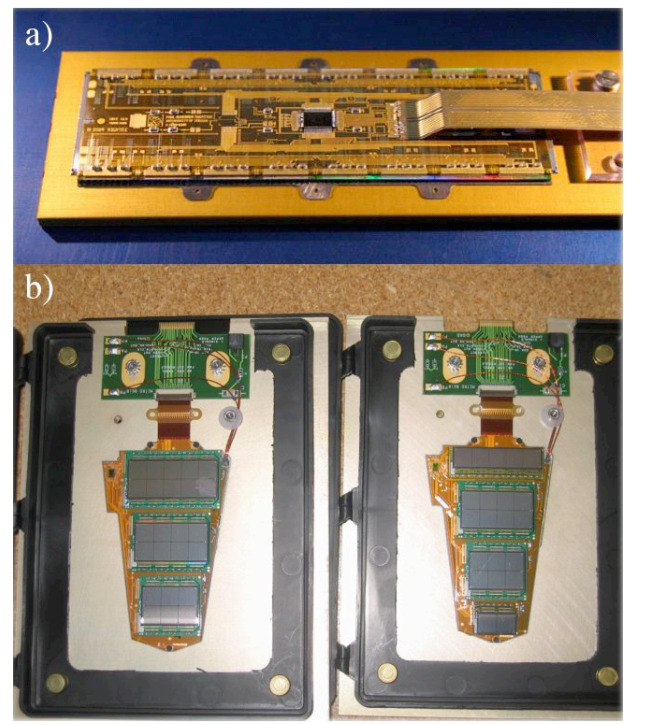

Fig. 3 Barrel modules (a) and end-cap plaquettes (b).

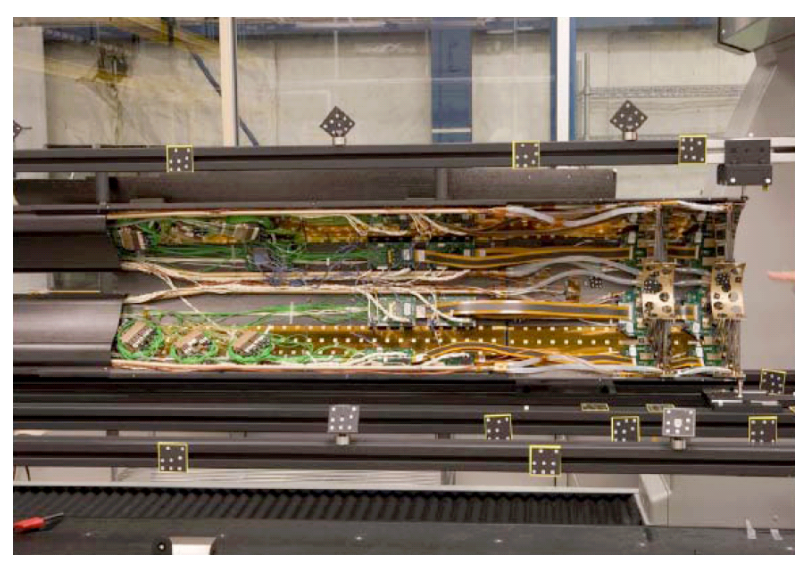

Fig. 4 View of one of the four half cylinder of the FPix detector. Two half disks are mounted in the final carbon fiber support structure. 